\title{
Nanofabrication at High Throughput and Low Cost
}

\author{
Benjamin J. Wiley, ${ }^{+, *}$ Dong Qin, ${ }^{*}$ and Younan $\mathrm{Xia}^{\mathrm{S}, *}$ \\ ${ }^{\dagger}$ Department of Chemistry, Duke University, Durham, North Carolina 27708, ${ }^{\ddagger}$ Department of Energy, Environmental and Chemical Engineering, Washington University, St. \\ Louis, Missouri 63130, and §Department of Biomedical Engineering, Washington University, St. Louis, Missouri 63130
}

\begin{abstract}
The task of nanofabrication can, in principle, be divided into two separate tracks: generation and replication of the patterned features. These two tracks are different in terms of characteristics, requirements, and aspects of emphasis. In general, generation of patterns is commonly achieved in a serial fashion using techniques that are typically slow, making this process only practical for making a small number of copies. Only when combined with a rapid duplication technique will fabrication at high-throughput and low-cost become feasible. Nanoskiving is unique in that it can be used for both generation and duplication of patterned nanostructures.
\end{abstract}

See the accompanying Article by Lipomi et al. on p 4017.

*Address correspondence to
bjw24@duke.edu, xia@biomed.wustl.edu.

Published online July 27, 2010. $10.1021 / \mathrm{nn} 101472 \mathrm{p}$

() 2010 American Chemical Society
1 he ability to pattern a material into small structures is essential to much of modern science and technology, with applications ranging from the production of integrated circuits, information storage devices, and display units to the fabrication of sensors, actuators, biochips, microfluidic devices, and micro-optical components. ${ }^{1}$ The practice of patterning is also known as lithography - a multiple-step process that typically begins with the design of a pattern in the form of a data set and ends with a patterned array of small features on the surface of a substrate. Depending on the application, the requirements for a successful lithographic process can vary substantially. The minimum feature size of a test pattern is usually the most obvious issue one must consider when selecting a proper lithographic technique. In microelectronics, for example, the growing demand for higher densities of integration, less power consumption, better performance, and reduction in cost has kept pushing the capability of photolithography down to the nanometer scale. The state-ofthe-art in high-throughput nanofabrication is a deep UV (at $193 \mathrm{~nm}$ ) photolithographic tool that utilizes liquid-immersion optics to pattern sub-50 $\mathrm{nm}$ structures across a 300 $\mathrm{mm}$ (12 in.) wafer. ${ }^{2}$ However, in many other applications outside of electronics, both cost and throughput could become more demanding parameters than the minimum feature size.

Generally speaking, a parallel process is better suited for high-throughput fabrication than a serial technique. Patterns are often generated using a serial technique and then transferred into multiple copies through a parallel process. The cost of a patterning process can be attributed to two major sources: (1) the capital and operating expenses associated with the tool itself, and (2) the specific environment (e.g., a cleanroom) required for operating the instrument. The cost is often a dominant factor in determining the availability of a nanofabrication tool. For example, the current deep UV lithographic tool has a remarkable throughput of 80 wafers $/ \mathrm{h}\left(2 \times 10^{-3} \mathrm{~m}^{2} / \mathrm{s}\right)$, but comes with the astonishing price tag of $\$ 30,000,000$. Certainly no university can afford to invest in such a tool, so academic researchers often have to rely on electronbeam lithography (EBL) for generating nanostructures. At prices ranging from $\$ 350,000$ for a typical scanning electron microscope to $\$ 2,000,000$ for a dedicated electron-beam writer, EBL is cheap only in comparison and has a much lower patterning throughput $\left(3 \times 10^{-10} \mathrm{~m}^{2} / \mathrm{s}\right.$ for an electron-beam writer). This nanofabrication tool is typically operated in a cleanroom, which is in itself expensive to construct and maintain. In addition, these conventional methods both require a smooth, rigid, and somewhat expensive substrate, which is most commonly based upon silicon at a price of $\$ 200-1000 / \mathrm{m}^{2}$, depending on the size and quantity.

There are many applications (both old and new), such as metamaterials, solar cells, sensors, actuators, and flexible electronics, which would benefit enormously from alternative methods of nanofabrication. Specifically, there is an increasing need to (1) pattern a wide variety of flexible substrates, (2) increase the throughput ( $\left.>1 \mathrm{~m}^{2} / \mathrm{s}\right)$, (3) move outside of the cleanroom, and (4) keep the capital investment and actual cost as low as possible. The tools and methods developed for microelectronics simply cannot meet these goals. Here, we discuss a number of promising approaches to nanofabrication that can reduce or eliminate the use of cleanrooms and associated tooling, can be applied to a variety of substrates, and have the potential to be applied for low-cost, high-throughput production. 


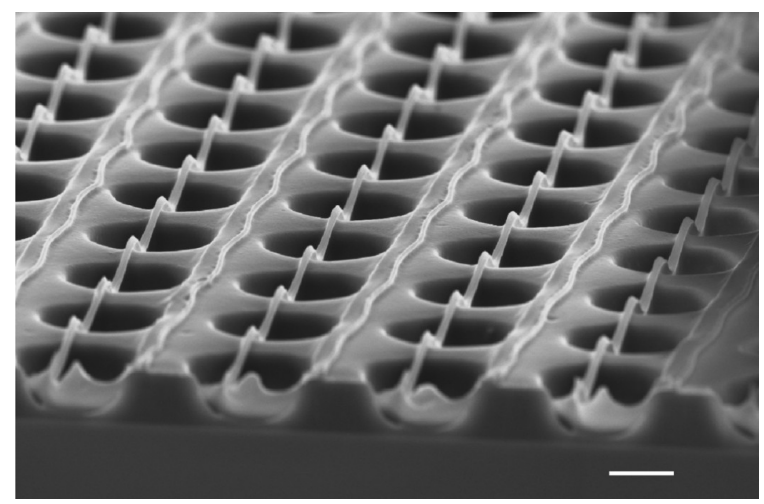

Figure 1. SEM image of a two-layered structure fabricated by molding with PDMS molds with a photosensitive polymer (SU8-2, for the bottom layer) and an e-beam sensitive polymer (PMMA, for the top layer). The scale bar is $2 \mu \mathrm{m}$. with soft, fluorocarbon stamps, as has been demonstrated by Kim et al. with their self-aligned imprint lithography (SAIL) process. ${ }^{6}$ With this improvement, they can pattern

Soft Lithography. This technology encompasses a number of different processes, including molding, printing, and embossing, that utilize an elastomeric stamp to transfer patterned features onto the surface of a substrate., ${ }^{3,4}$ Stamps with nanoscale features must be molded by casting a curable prepolymer onto a master, which is typically fabricated using EBL. In this case, one must either have access to EBL to make a master or order a master from a commercial source (e.g., from www.nilt.com). Subsequent patterning with the stamp can be performed outside of the cleanroom; debris on the master is usually taken up by the stamp after casting. Stamps can also be cleaned with masking tape.

After generation of a stamp, nanoscale patterns can be formed on a surface by either printing, molding, or embossing. In an embossing process, a solvent is used to soften a polymer, the stamp is pressed into the polymer, and the solvent is then allowed to dry before removal of the stamp. Line widths of $60 \mathrm{~nm}$ have been demonstrated with this process, and it can also be used to generate multilayered structures in a layer-by-layer fashion (Figure 1). ${ }^{5}$ A factor that limits the throughput of this technique is the requirement that the stamp must be in contact with the polymer while the organic solvent evaporates. This can be overcome by molding photocurable polymers features as small as $100 \mathrm{~nm}$ at a rate of $2.8 \times 10^{-2} \mathrm{~m}^{2} / \mathrm{s}$ (Figure 2).

For printing with soft stamps, the ink can either be molecular in nature (e.g., alkanethiols, proteins, and dendrimers, among others) or be a solid material (e.g., nanoparticles, solid films). ${ }^{7-11}$ Generally speaking, it seems to be easier to generate nanostructures by printing solids because molecular inks can diffuse across a surface, and the transfer of a molecular ink from a stamp to a substrate is more difficult to control. That being said, researchers have demonstrated generation of sub-50 $\mathrm{nm}$ lines by printing high molecular weight inks. ${ }^{12}$ Lines of nanoparticles down to $67 \mathrm{~nm}$ in diameter can be printed by careful control of the assembly of the nanoparticles on the stamp. ${ }^{13}$

Although the minimum resolution of soft lithography is not quite as good as the other techniques discussed below, soft stamps can (1) generate features over large, curved surfaces; (2) print a rich variety of materials on a wide range of substrates; and (3) be used in roll-to-roll processes.

Imprinting. Of the methods that have been developed, nanoimprint lithography (NIL) is probably the closest to commercial viability. Tools available from Molecular Imprints for manufacturing hard-disk drives advertise sub-20 nm resolution and throughputs of 300 disks/ h. ${ }^{14}$ This tool utilizes a hard, clear stamp (made of quartz) to mold a low-viscosity, photocurable monomer solution. This method, termed "step and flash" imprint lithography (S-FIL), can generate lines with a 6 $\mathrm{nm}$ half-pitch (see Figure 3) with high pattern fidelity (for $20 \mathrm{~nm}$ lines, standard deviation is \pm 1.2 $\mathrm{nm}) .{ }^{15}$ Three-dimensional structure can also be generated (e.g., T-gate, damascene interconnect), thereby saving processing steps, and a full wafer can be patterned simultaneously. One of the major challenges with this method is fouling of and damage to the stamp, which is rather slow and expensive to fabricate. Thus, S-FIL must be car-
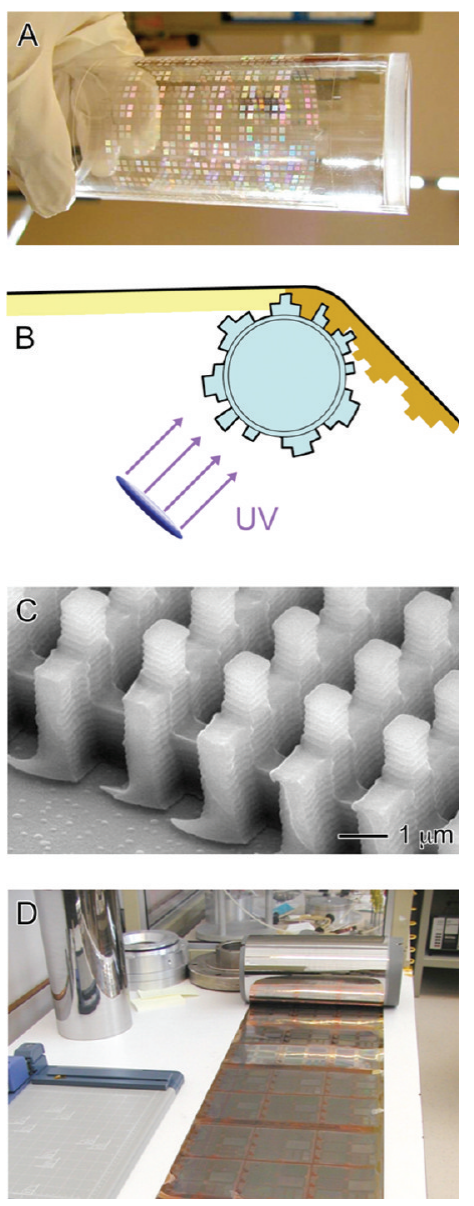

Figure 2. (A) Elastomeric imprint stamp wrapped around a UVtransparent quartz roller. (B) UV light passes through the roller to cure the imprint polymer. (C) Scanning electron microscope image of submicrometer features imprinted at a roller speed of $5 \mathrm{~m} / \mathrm{min}$. (D) Active matrix backplanes for displays, completely fabricated in a roll-to-roll process on a roll $0.3 \mathrm{~m}$ wide. Reproduced with permission from ref 6. Copyright 2009 Society for Information Display. 


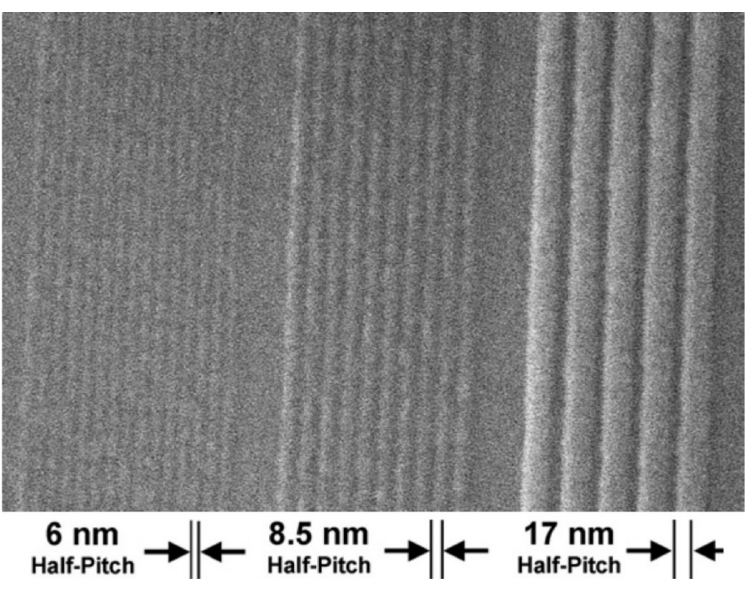

Figure 3. Scanning electron microscope image of a UV-curable polymer resist patterned with $6,8.5$, and $17 \mathrm{~nm}$ half-pitch gratings. Reproduced with permission from ref 15. Copyright 2005 Institute of Physics Publishing.

ried out in a cleanroom environment, and the expensive stamps make this technique less attractive and accessible to researchers in academia. Like photolithography, S-FIL is designed for and well-suited to fabrication in the semiconductor industry, but it may not be suitable for high-throughput production in a roll-to-roll process.

\section{Of the methods that}

have been developed, nanoimprint

\section{lithography is probably \\ the closest to}

\section{commercial viability.}

Thermal-NIL likely has greater potential for high-throughput, lowcost patterning. This method, which is essentially hot embossing, can use relatively inexpensive metal stamps on rollers to create sub-100 $\mathrm{nm}$ features in a polymer. ${ }^{16}$ The throughput of this process, $4 \times$ $10^{-4} \mathrm{~m}^{2} / \mathrm{s}$, is lower than soft lithography. Thermal-NIL has demonstrated features as small as $10 \mathrm{~nm}$, and patterning of an entire wafer at once is possible. ${ }^{17}$ Because the substrate must be very smooth to make good contact with the mold, the range of substrates that can be patterned is more limited than the other three processes discussed here. The lower cost of the mold and relative simplicity of the process makes thermal-NIL a good option for academic researchers who require smaller features than is possible with soft lithography and over larger areas than is possible with EBL.

Nanoskiving. This technique combines thin-film deposition with sectioning to generate nanostructures. ${ }^{18}$ It involves two major steps: (1) embedding a thin metallic, semiconducting, or polymeric film in an epoxy block; and (2) sectioning the block into slabs (10-30 nm thick) with an ultramicrotome. Sectioning a thin film (as thin as $10 \mathrm{~nm}$ ) generates a nanowire $(10-30 \mathrm{~nm}$ in lateral dimensions) that can be millimeters in length. By starting with a thin film deposited on a topographically patterned substrate, nanoskiving can generate a wide variety of nanostructures that would otherwise be difficult or impossible to fabricate (Figure 4). Sectioning of polymer multilayers formed by sequential spin coating resulted in an array of polymer nanowires that could act as an ordered bulk heterojunction solar cell. ${ }^{19}$ Single-crystal nanostructures prepared using a bottom-up approach could be carved by nanoskiving to generate nanostructures of great interest to plasmonics. ${ }^{20}$ It should be pointed out that epoxy slabs containing the nanostructures can be readily deposited on virtually any substrate. Magnetic particles can also be co-embedded within the slab to help control its placement on a surface with an accuracy of $13 \mu \mathrm{m} .^{21}$

At a price of $\$ 60,000$, the instrument required for nanoskiving, the ultramicrotome, is relatively inexpensive. Nanoskiving also requires investment in a diamond knife ( \$2000) that must be sharpened

\section{Nanoskiving can}

generate a wide variety

of nanostructures that

would otherwise be

difficult or impossible

to fabricate.

every 6-12 months at a cost of $\sim$ \$1000. These expenses represent a miniscule investment relative to the cost of a cleanroom and the associated tools. The throughput of nanoskiving $\left(2.8 \times 10^{-7} \mathrm{~m}^{2} / \mathrm{s}\right)$ is still relatively low compared to techniques that have had a longer time to develop. Commercially available ultramicrotomes are not well-suited for high-volume production because each slab must be collected by hand from a pool of water, and the area per slice is small $\left(\sim 1 \mathrm{~mm}^{2}\right)$. A recently developed, automated tape-collecting ultramicrotome represents a first step toward highthroughput nanoskiving.

Even without the capability of high-throughput production, nanoskiving is a very useful and underutilized tool for researchers who want to study the properties of nanostructures. The work by Lipomi et al. in this issue highlights the unique capabilities of nanoskiving as they demonstrate the ability to generate metallic nanostructures (e.g., concentric crescents of gold) of relevance to metamaterials over a $1 \mathrm{~mm}^{2}$ area. ${ }^{22}$ Photolithography and soft lithography cannot generate these structures, and fabricating them by EBL or focused ion beam (FIB) milling over the same area would be very costly and timeconsuming. Additional advantages of nanoskiving for this application include the abilities to (1) place the nanostructures on any substrate, (2) generate gradients of height, (3) generate three-dimensional structures easily, (4) quickly generate 
multiple copies, and (5) generate crescents in close proximity with different dielectric materials. The key advances that enable this fabrication include (1) the use of EBL, reactive ion etching (RIE), and replica molding to generate an array of nanoscale posts with high aspect ratios in epoxy; and (2) controlled deposition of materials on the nanoscale posts by shadow evaporation.

Whereas the work by Lipomi et al. was mainly focused on making circular rings from posts, we note that the same process can generate nanostructures complementary to any array of structures generated by EBL. As a simple example, by using square instead of circular features as the mask for RIE, one could generate an array of square rings. Thus the work by Lipomi et al. represents an exciting opportunity for further exploration of the use of nanoskiving to generate arrays of multicomponent nanostructures in arbitrary patterns on any substrate.

Self-Assembly. With life as the ultimate example, self-assembly offers the greatest potential for mass production of nanostructures. ${ }^{23} \mathrm{Al}-$ though we see the potential for selfassembly all around us, it has proven staggeringly difficult to gain a comparable level of control over selforganization in the lab. Only within the past few years have researchers learned to program life's hard-drive, DNA, to control the assembly of nanostructures. ${ }^{24}$ Exciting recent developments include control over the assembly of carbon nanotubes into crossbars, DNA robots, and a variety of DNA structures than controllably respond to inputs. ${ }^{25-28}$ Given the cost and time required to synthesize DNA and the necessity of patterning it on a surface, it will be some time before recent developments are translated into viable options for high-throughput nanofabrication.

Block copolymers have had relatively more time to be incorporated into a practical method for sub-30 nm nanofabrication. ${ }^{29}$ Hexagonal arrays of cylindrical pores formed in block copolymer films are being developed as etch masks to generate low- $k$ dielectrics for transistor gates. $^{30}$ Patterning of magnetic storage media is another potential application for the arrayed holes. Spontaneous assembly of square arrays has also been demonstrated, but patterning of more complicated structures (e.g., lines) still

\section{With life as the ultimate}

example, self-assembly

offers the greatest

\section{potential for mass}

\section{production of}

\section{nanostructures.}

requires a lithographically defined template. ${ }^{31-33}$ Nanofabrication with block copolymers is a very accessible method for academic researchers to generate arrays of quantum dots or nanowires on a surface. However, the fact that block copolymer films may take several days to anneal is a significant barrier to their application for high-throughput nanofabrication.

In the near term, colloidal nanostructures and their assemblies offer the greatest potential for commercialization. Inks composed of metallic nanoparticles are already used commercially to generate conducting structures for flexible electronics produced in roll-to-roll
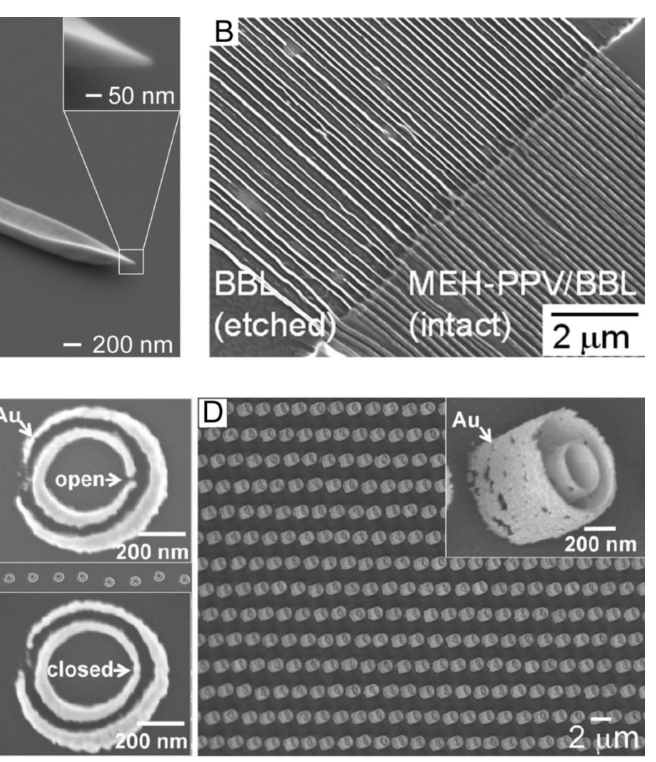

00000000000000000002 2 um

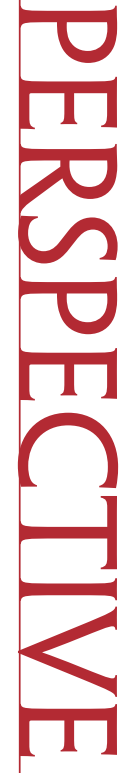


print lithography to reduce the minimum feature size further. At the same time, the potential for economic gain with smaller, more powerful chips is dwarfed by the market for mobile, rugged, cheap, ubiquitous, and eco-friendly sensors, electronics, and power sources. Soft lithography is providing a key enabling technology in the development of roll-to-roll processes to drive down the cost of these devices. Nanoskiving and selfassembly have the potential to generate smaller and more complex features than is possible with soft lithography, but further research is required before they become economically viable nanofabrication technologies. The demonstration by Lipomi et al. in this issue of the potential of nanoskiving to generate copies of arbitrary arrays of multicomponent nanostructures on virtually any substrate will provide an impetus for other researchers to accelerate the development of this technique, especially in the context of metamaterials. Although a great deal of work has been done with self-assembly, which has the greatest untapped potential for highthroughput nanofabrication, we are still at the embryonic stage of understanding and balancing nanoscale forces to drive the assembly of materials into device architectures.

It is not easy to predict the directions that a technology might take in the future. Experts in the field of electronics, for example, speculated many times that the end of photolithography was just around the corner. However, quite the opposite has occurred, and this technology remains more vital than ever. The transition to shorter wavelengths and clever implementation of resolution-enhancement techniques (e.g., phase shift and immersion optics) have kept pushing the physical limits set by optical diffraction, and this trend is now expected to continue at least for the foreseeable future. On the contrary, many techniques (e.g., $\mathrm{EBL}$ and X-ray lithography) that had been forecasted to provide alternative solutions to photolithography showed more severe challenges than were anticipated. Indeed, there seems to be no obvious replacement for photolithography despite the tremendous efforts put forth in establishing new, alternative technologies. These developments, however, have always spurred significant advances in other, sometimes not obviously related, fields such as physics, chemistry, materials science, and optics. There is no doubt that such synergies will further evolve and continue to be a rich source of inspiration and surprise for both scientific research and technological development.

Acknowledgment. During the preparation of this article, Y.X. was partially supported by the WCU (World Class University) program through the National Research Foundation of Korea, funded by the Ministry of Education, Science and Technology (R32-20031).

\section{REFERENCES AND NOTES}

1. Geissler, M.; Xia, Y. N. Patterning: Principles and Some New Developments. Adv. Mater. 2004, 16, 1249-1269.

2. Pease, R. F.; Chou, S. Y. Lithography and Other Patterning Techniques for Future Electronics. Proc. IEEE 2008, 96, 248-270.

3. Xia, Y. N.; Whitesides, G. M. Soft Lithography. Annu. Rev. Mater. Sci. 1998, 28, 153-184.

4. Qin, D.; Xia, Y. N.; Whitesides, G. M. Soft Lithography for Micro- and Nanoscale Patterning. Nat. Protoc. 2010, 5, 491-502.

5. Kim, E.; Xia, Y. N.; Zhao, X. M.; Whitesides, G. M. Solvent-Assisted Microcontact Molding: A Convenient Method for Fabricating Three-Dimensional Structures on Surfaces of Polymers. Adv. Mater. 1997, 9, 651-654.

6. Kim, H. J.; Almanza-Workman, M.; Garcia, B.; Kwon, O.; Jeffrey, F.; Braymen, S.; Hauschildt, J.; Junge, K.; Larson, D.; Stieler, D.; et al. Roll-to-Roll Manufacturing of Electronics on Flexible Substrates Using Self-Aligned Imprint Lithography (SAIL). J. Soc. Inf. Disp. 2009, 17, 963-970.

7. Rogers, J. A.; Bao, Z.; Baldwin, K.; Dodabalapur, A.; Crone, B.; Raju, V. R.; Kuck, V.; Katz, H.; Amundson, K.; Ewing, J.; et al. Paper-like Electronic Displays: Large-Area Rubber-Stamped Plastic Sheets of Electronics and Microencapsulated
Electrophoretic Inks. Proc. Natl. Acad. Sci. U.S.A. 2001, 98, 4835-4840.

8. Kane, R. S.; Takayama, S.; Ostuni, E.; Ingber, D. E.; Whitesides, G. M. Patterning Proteins and Cells Using Soft Lithography. Biomaterials 1999, 20, 2363-2376.

9. Bernard, A.; Renault, J. P.; Michel, B.; Bosshard, H. R.; Delamarche, E. Microcontact Printing of Proteins. Adv. Mater. 2000, 12, 1067-1070.

10. Perl, A.; Reinhoudt, D. N.; Huskens, J. Microcontact Printing: Limitations and Achievements. Adv. Mater. 2009, 21, 2257-2268.

11. Zaumseil, J.; Meitl, M. A.; Hsu, J. W. P.; Acharya, B. R.; Baldwin, K. W.; Loo, Y. L.; Rogers, J. A. ThreeDimensional and Multilayer Nanostructures Formed by Nanotransfer Printing. Nano Lett. 2003, 3, 1223-1227.

12. Li, H. W.; Muir, B. V. O.; Fichet, G.; Huck, W. T. S. Nanocontact Printing: A Route to Sub-50-nm-Scale Chemical and Biological Patterning. Langmuir 2003, 19, 1963-1965.

13. Kraus, T.; Malaquin, L.; Schmid, H.; Riess, W.; Spencer, N. D.; Wolf, H. Nanoparticle Printing with SingleParticle Resolution. Nat. Nanotechnol. 2007, 2, 570-576.

14. Costner, E. A.; Lin, M. W.; Jen, W. L.; Willson, C. G. Nanoimprint Lithography Materials Development for Semiconductor Device Fabrication. Annu. Rev. Mater. Sci. 2009, 39, 155-180.

15. Austin, M. D.; Zhang, W.; Ge, H. X.; Wasserman, D.; Lyon, S. A.; Chou, S. Y. $6 \mathrm{~nm}$ Half-Pitch Lines and 0.04 $\mu \mathrm{m}^{2}$ Static Random Access Memory Patterns by Nanoimprint Lithography. Nanotechnology 2005, 16, 1058-1061.

16. Tan, H.; Gilbertson, A.; Chou, S. Y. Roller Nanoimprint Lithography. J. Vac. Sci. Technol., B 1998, 16, 3926-3928.

17. Chou, S. Y.; Krauss, P. R.; Zhang, W.; Guo, L.; Zhuang, L. Sub-10 nm Imprint Lithography and Applications. J. Vac. Sci. Technol., B 1997, 6, 2897-2904.

18. Xu, Q. B.; Rioux, R. M.; Dickey, M. D.; Whitesides, G. M. Nanoskiving: A New Method To Produce Arrays of Nanostructures. Acc. Chem. Res. 2008, 41, 1566-1577.

19. Lipomi, D. J.; Chiechi, R. C.; Reus, W. F.; Whitesides, G. M. Laterally Ordered Bulk Heterojunction of Conjugated Polymers: Nanoskiving a Jelly Roll. Adv. Funct. Mater. 2008, 18, 3469-3477.

20. Wiley, B. J.; Lipomi, D. J.; Bao, J. M.; Capasso, F.; Whitesides, G. M. Fabrication of Surface Plasmon Resonators by Nanoskiving SingleCrystalline Gold Microplates. Nano Lett. 2008, 8, 3023-3028.

21. Lipomi, D. J.; llievski, F.; Wiley, B. J.; Deotare, P. B.; Loncar, M.; 
Whitesides, G. M. Integrated Fabrication and Magnetic Positioning of Metallic and Polymeric Nanowires Embedded in Thin Epoxy Slabs. ACS Nano 2009, 3, 3315-3325.

22. Lipomi, D. J.; Kats, M. A.; Kim, P.; Kang, S. H.; Aizenberg, J.; Capasso, F.; Whitesides, G. Fabrication and Replication of Arrays of Single- or Multi-Component Nanostructures by Replica Molding and Mechanical Sectioning. ACS Nano 2010, 4 4017-4026.

23. Whitesides, G. M.; Grzybowski, B. Self-Assembly at All Scales. Science 2002, 295, 2418-2421.

24. Becerril, H. A.; Woolley, A. T. DNATemplated Nanofabrication. Chem. Soc. Rev. 2009, 38, 329-337.

25. Lund, K.; Manzo, A. J.; Dabby, N.; Michelotti, N.; Johnson-Buck, A.; Nangreave, J.; Taylor, S.; Pei, R. J.; Stojanovic, M. N.; Walter, N. G.; et al. Molecular Robots Guided by Prescriptive Landscapes. Nature 2010, 465, 206-210.

26. Maune, H. T.; Han, S. P.; Barish, R. D.; Bockrath, M.; Goddard, W. A.; Rothemund, P. W. K.; Winfree, E. Self-Assembly of Carbon Nanotubes into Two-Dimensional Geometries Using DNA Origami Templates. Nat. Nanotechnol. 2010, 5, 61-66.

27. Andersen, E. S.; Dong, M. D.; Nielsen, M. M.; Jahn, K.; LindThomsen, A.; Mamdouh, W.; Gothelf, K. V.; Besenbacher, F.; Kjems, J. DNA Origami Design of Dolphin-Shaped Structures with Flexible Tails. ACS Nano 2008, 2 , 1213-1218.

28. Andersen, E. S.; Dong, M.; Nielsen, M. M.; Jahn, K.; Subramani, R.; Mamdouh, W.; Golas, M. M.; Sander, B.; Stark, H.; Oliveira, C. L. P.; et al. Self-Assembly of a Nanoscale DNA Box with a Controllable Lid. Nature 2009, 459, 73-76.

29. Fasolka, M. J.; Mayes, A. M. Block Copolymer Thin Films: Physics and Applications. Ann. Rev. Mater. Res. 2001, 31, 323-355.

30. Hermans, T. M.; Choi, J.; Lohmeijer, B. G. G.; Dubois, G.; Pratt, R. C.; Kim, H. C.; Waymouth, R. M.; Hedrick, J. L. Application of Solvent-Directed Assembly of Block Copolymers to the Synthesis of Nanostructured Materials with Low Dielectric Constants. Angew. Chem., Int. Ed. 2006, 45, 6648-6652.

31. Tang, C. B.; Lennon, E. M.; Fredrickson, G. H.; Kramer, E. J.; Hawker, C. J. Evolution of Block Copolymer Lithography to Highly Ordered Square Arrays. Science 2008, 322, 429-432.

32. Chai, J.; Wang, D.; Fan, X. N.; Buriak, J. M. Assembly of Aligned Linear Metallic Patterns on Silicon. Nat Nanotechnol. 2007, 2, 500-506.

33. Stoykovich, M. P.; Muller, M.; Kim, S. O.; Solak, H. H.; Edwards, E. W.; de
Pablo, J. J.; Nealey, P. F. Directed Assembly of Block Copolymer Blends into Nonregular DeviceOriented Structures. Science 2005, 308, 1442-1446.

34. Gamerith, S.; Klug, A.; Scheiber, $\mathrm{H}_{\text {; }}$ Scherf, U.; Moderegger, E.; List, E. J. W. Direct Ink-Jet Printing of $\mathrm{Ag}-\mathrm{Cu}$ Nanoparticle and AgPrecursor Based Electrodes for OFET Applications. Adv. Funct. Mater. 2007, 17, 3111-3118.

35. Krebs, F. C. Polymer Solar Cell Modules Prepared Using Roll-toRoll Methods: Knife-over-Edge Coating, Slot-Die Coating and Screen Printing. Sol. Energy Mater. Sol. Cells 2009, 93, 465-475.

36. Rogers, J. A.; Paik, U. Nanofabrication: Nanoscale Printing Simplified. Nat. Nanotechnol. 2010, 5, 385-386.

37. Wang, M. C. P.; Gates, B. D. Directed Assembly of Nanowires. Mater. Today 2009, 12, 34-43.

38. Hu, L. B.; Kim, H. S.; Lee, J. Y.; Peumans, P.; Cui, Y. Scalable Coating and Properties of Transparent, Flexible, Silver Nanowire Electrodes. ACS Nano 2010, 4, 2955-2963.

39. Rathmell, A. R.; Bergin, S. M.; Hua, Y.-L.; Li, Z.-Y.; Wiley, B. J. The Growth Mechanism of Copper Nanowires and Their Properties in Flexible, Transparent Conducting Films. Adv. Mater. 2010, DOI: 10.1002/adma.201000775.

40. See http://www.cambrios.com/ for a commercial source of transparent conductive, flexible sheets based on silver nanowires. 